\title{
Un itinerario de investigación sobre vivienda social y calidad de vida. Reflexión metodológica desde la geografía humana
}

Research itinerary on social housing and quality of life. A methodological reflection from human geography

\author{
Fabricio Espinosa Ortiz \\ CONACYT - Consorcio CentroMet, México \\ fabricio.espinosa@centromet.mx
}

\section{Resumen:}

Este trabajo tiene dos objetivos interrelacionados: el primero consiste en explorar, el itinerario metodológico de una investigación que comprendió el impacto que tiene la vivienda social en la calidad de vida de los habitantes de la periferia metropolitana. El segundo objetivo es articular, a partir del itinerario, reflexiones sobre la vivienda social y la calidad de vida en las periferias metropolitanas, desde la perspectiva de la disciplina de la Geografía Humana. Se finaliza destacando que la calidad de vida no es estática ni en el espacio ni el tiempo, y la metodología para su estudio en relación con la vivienda requiere de la flexibilidad que integre las relaciones e interacciones entre las dimensiones materiales e inmateriales, individuales y colectivas, y multiescalares de los territorios habitados.

Palabras Clave: Itinerario de investigación, Vivienda social, Calidad de vida, Geografía humana.

\section{Abstract:}

This paper has two interrelated goals. First, it explores the methodological itinerary of a study that analyzed the impact of social housing on periphery's inhabitants' quality of life. Second, from that itinerary, it reflects on social housing and quality of life in metropolitan peripheries, from the perspective of Human Geography. It concludes that quality of life is not static, neither in space or time; therefore, the methodology for its study of housing must allow the necessary flexibility to integrate relations and interactions between such dimensions of the inhabited territories as material and immaterial, individual and collective, and multiscale.

KEYWORDS: Methodological itinerary, Social housing, Quality of life, Human geography.

\section{INTRODUCCIÓN}

En las últimas décadas las ciencias sociales han sido partícipes de un conjunto de transformaciones teóricas y metodológicas en las que también ha estado inmersa la geografía humana. Estas transformaciones representan giros disciplinarios en los cuales se reconoce que la sociedad es producida y reproducida por los sujetos (Giddens, 1995; Elias, 1990). En el caso de la geografía, esto constituye su apertura como la disciplina que tradicionalmente había estado orientada por enfoques objetivistas y/o materialistas a incluir en la conceptualización de su principal objeto de estudio, la relación espacio-sociedad, a lo inmaterial e intangible, para redescubrir al sujeto y su subjetividad en sus experiencias espaciales (Seamon, 1979; García Ramón, 1999; Philo, 1999; Warf y Arias, 2008), lo cual fortalece el dialogo del quehacer geográfico con otras ciencias sociales, como la lingüística, la sociología, la psicología y la antropología (Lindón y Hiernaux, 2010).

Lo anterior ha traído un intenso interés por el estudio de las relaciones del sujeto en el espacio y con el espacio en su sentir, percibir y concebir los lugares que transita y habita cotidianamente, lo que impulsa a la Geografía al estudio de las articulaciones entre la materialidad del espacio con el sujeto y su subjetividad como la adecuada estrategia para entender mejor las formas de habitar los diferentes territorios. En este caso se estudia la ciudad como lugar de complejidad de la vida urbana, que en buena medida se despliega en ella por los volúmenes crecientes de población en condición de pobreza, por la concentración de poder y sin poder 
y las consecuentes ventajas y desventajas sociales a las que se enfrentan los habitantes, no obstante, todos partícipes de la producción y reproducción de la ciudad, que es resultado de la obra constante de ellos mismos (Lindón, 2009).

Por otra parte, cabe señalar que los estudios de la ciudad desde la Geografía han ampliado su interés por las escalas espaciales más próximas al habitante. El mejor ejemplo es el análisis de la vivienda y su entorno inmediato (fraccionamientos, colonias, barrios, etcétera), el cual se consideraba anteriormente una escala de análisis del quehacer del arquitecto (Blunt, 2005; Blunt y Dowling, 2006; Brickell, 2012; Collignon, 2010; Lindón, 2005 y 2006; Mallet, 2004). Este giro de la Geografía busca superar las visiones tradicionales del espacio racional cartesiano, así como el predominio de los enfoques de análisis de los grandes territorios, para atender también el punto de vista del sujeto que los habita (Hiernaux, 2008), indagarlos en relación con la continua e inacabada construcción de sus lugares y las prácticas sociales cotidianas que se despliegan en ellos y por ellos (Cresswell, 2004, p. 37).

Desde esta perspectiva, en el trabajo se aborda el estudio de la vivienda social a partir del análisis del habitar cotidiano debido a su capacidad para dar cuenta de la relación del sujeto con los lugares. Se plantean dos objetivos centrales: el primero consiste en mostrar, a partir de un caso, el itinerario metodológicoconceptual de una investigación que buscó determinar el impacto que tiene la vivienda social (denominada en México como vivienda de interés social), (SEDATU, CONAVI, 2017: 61), localizada en la periferia suroeste de la ciudad de Morelia, Michoacán, México, en la calidad de vida de sus habitantes, en la escala del fraccionamiento ${ }^{1}$, y en relación con la ciudad. El segundo objetivo es articular, a partir del análisis de esta porción de la ciudad, reflexiones en torno a los significados que los usuarios depositan en su vivienda y fraccionamiento, además de lo que condiciona su calidad de vida en ese lugar. Para ello, resultó central el reconocimiento desde nuestro modelo explicativo de investigación, explicar que la vivienda, más allá de ser una unidad física material que acoge a una familia, es un sistema de escalas espaciales que se relacionan entre sí, y que incluyen, además de la vivienda en su estructura, su entorno inmediato y contexto urbano. Este sistema de escalas da continuidad a la acción de habitar de sus residentes, y define a la vivienda como una unidad integrada al entorno, escenario cotidiano de las relaciones e interacciones sociales, depositario y generador de significados, emociones, deseos y aspiraciones diversas (Cortés, 1996; Espinosa, 2015).

Por último, cabe destacar que en este texto no se presentan datos duros, ya que se hace una interpretación de nuestro proceso de producción de información procedente del trabajo de campo realizado (entrevistas, recorridos, observación participante y aplicación de cuestionarios en la zona de estudio) ${ }^{2}$. Las reflexiones se dan a la luz de nuestra propia experiencia en la investigación, así que la intención de este texto es presentar las bases teóricas y epistemológicas del método de investigación, y con ello tratar de delinear algunos retos metodológicos en el estudio de la vivienda en las periferias metropolitanas, y de la calidad de vida de quienes las habitan. El estudio del territorio periférico se hizo, pues, desde la perspectiva de los habitantes; esto, con la intención de abordajes metodológicos anclados en las estrategias prácticas de los actores, con un importante énfasis en el desarrollo de trabajo de campo que supone la combinación de diversas estrategias cualitativas de investigación social. Consideramos que del conjunto, resulta una contribución significativa que podría ser complementada, en instancias a posteriori, con el desarrollo de una mirada comparada darles voz a través de sus narrativas de habitar. Colocar nuestra mirada en el habitar nos brinda la posibilidad de indagar en las cualidades que puede tener el espacio en la experiencia de vivirlo y en los significados que para los habitantes de la periferia tiene su vivienda, lo que implica en sí mismo hacerlos visibles, y, a partir de eso, dilucidar la valoración que le otorgan a su vida en ese lugar.

\section{ENFOQUE TEÓRICo METODOLÓGICo}

Partimos de la premisa de que la visión de los arquitectos, urbanistas, planificadores y/o promotores de los conjuntos habitacionales es ajena a la realidad del sujeto que los habita y que valora sus propias condiciones de vida en estos entornos. Esta forma de ver la ciudad se denomina exocéntrica (Hiernaux y Lindón, 2004), y es entendida como aquella en la que los especialistas, analistas y profesionales ofrecen 
una interpretación del fenómeno a partir de su propia concepción y experiencia; la visión exocéntrica es periférica, es externa, es realizada desde afuera, desde el escritorio o a vuelo de pájaro. Las visiones exocéntricas se construyen desde los imaginarios de cada época, como el de vivir bien, el de felicidad, el del progreso, el de calidad de vida, entre otros que tienen que ver con las fuerzas del mercado inmobiliario y las estrategias de los planificadores, promotores inmobiliarios, urbanistas, políticos y servidores públicos, para hacer concordancia con estas fuerzas e impulsarlas. Otra forma de ver la ciudad se denomina egocéntrica (Hiernaux y Lindón, 2004), y parte de la interpretación del habitante como protagonista de su propio habitar; esta forma de ver la ciudad también se puede realizar por los especialistas, que interpretan la interpretación del habitante, es decir, buscan desentrañar la ciudad desde el punto de vista de quienes la habitan. En el caso de nuestra investigación, comprender el habitar desde adentro, con sus condiciones y condicionantes, permitió tener los elementos de análisis para dilucidar cómo valoran los habitantes su calidad de vida en sus entornos habitacionales. La visión exocéntrica en el estudio de la ciudad está mucho más desarrollada que la egocéntrica, pero no solo en el estudio, sino también en la práctica del urbanismo y la planificación urbana-territorial, ya que las políticas e intervenciones urbanas son guiadas por la rentabilidad del suelo y el escenario político; y no se toman en cuenta las necesidades de los habitantes en las decisiones de los proyectos urbanos (Cuenya, 2012, pp. 1020-1022). En consecuencia, proyectos urbanos como los de los grandes conjuntos habitacionales nunca han formado parte de una alternativa de urbanismo redistributivo, ni menos aún de un urbanismo ciudadano (Silva, 2013, pp. 159-162); solo benefician a un sector muy reducido de la población, ya que es escasa la recuperación de plusvalías para la ciudad y las enormes ganancias son concentradas por los promotores inmobiliarios. Por ello, se conforma así un urbanismo sin ciudad, caracterizado por la suma de diferentes urbanizaciones aisladas, fragmentadas, con tendencia al desuso, y otras, por el contrario, con tendencia al uso intensivo. Esta contrariedad forma parte del costo que hay que pagar por la escala urbana (Borja, 2017, p. 4).

Ante estos fenómenos de la expansión exacerbada de las periferias metropolitanas, la disponibilidad de suelo se limita, lo que produce alzas en los precios de la tierra, y, por consiguiente, de las viviendas en localizaciones más cercanas a los equipamientos y servicios de las ciudades. Con ello, la oferta de vivienda social para la población de menores recursos se da en conjuntos habitacionales cada vez más alejados, lo que dificulta para los gobiernos locales el abastecimiento de equipamientos, espacios públicos y servicios a los habitantes (Ziccardi, 2016, pp. 13-16). Ante esta situación, los ciudadanos no solo se ven obligados a asumir altos costos económicos en sus traslados, sino también los relacionados con la resignación de habitar en un entorno habitacional con déficits en equipamientos y servicios, que además propician la acumulación de otros déficits, como el de ciudadanía e integración social (Borja, 2017).

Si la calidad de vida, según Diener (2006), se refiere al grado en que la vida de una persona es deseable por un grupo social en comparación con las que se consideran indeseables, el análisis del ingreso, el empleo, la educación, resultan insuficientes, ya que la comparación social y la valoración de la vida incluyen experiencia subjetiva, y, con ello, circunstancias de vida, percepciones, emociones, sentimientos y reacciones a las circunstancias. Es el ser humano quien está en el centro de la calidad de vida, ya que es él quien percibe, soporta, articula y da sentido a la acción y a la vida, y, por lo tanto, es él quien la valora de una forma u otra (Allardt, 1996, pp. 126-134; Scanlon, 1996, pp. 245-263). El habitante aprende cuáles son los obstáculos y las potencialidades de los espacios que habita en relación con los acontecimientos sucedidos y las experiencias vividas en los mismos, así como en la medida en que aprende a vivir en ese lugar (Tuan, 2007, pp. 92-95). Los habitantes son quienes ponen en práctica sus tácticas (De Certeau, 1996) para satisfacer sus necesidades cotidianas, para sortear obstáculos, así como para sobrellevar sus miedos y demás emociones que influyen en la valoración que hacen sobre su calidad de vida. Por lo anterior, se considera insuficiente para el estudio de la calidad de vida recurrir a la predeterminación de indicadores y a la correlación simplista de variables, y/o recoger datos sobre el perfil de la población (edades, profesiones, ingresos, etcétera), ya que así se dejan 
de lado las interpretaciones que hacen las personas sobre su vida. Los criterios tradicionales de medición de la calidad de vida tienden a reducir la complejidad de la vida a simples variables haciendo alusión a sujetos iguales, y no consideran que la valoración por la vida no se produce en abstracto, y que solo tiene sentido con respecto a la forma de vida de cada persona, sus sentimientos, su familia, sus relaciones sociales, su trabajo, sus intereses, etcétera.

De acuerdo con lo anterior, se plantea que en la evaluación de la calidad de vida no existe un modelo único y englobante, y que es necesario reconocer las aspiraciones, experiencias y satisfacciones que tienen los habitantes en relación con el entorno espacial en que se encuentran inmersos. En consecuencia, en esta investigación, más que delimitar los indicadores de medición con precisión absoluta, se asumió trabajar con conceptos sensibilizadores, es decir, con categorías densas e integradoras, que, en vez de aislar y seccionar, integraran y representaran direcciones en las cuales podemos concentrar la observación en nuestra búsqueda e interpretación de la información a lo largo del proceso de investigación (Blumer, 1969).

Se consideró que sería equivocado ver las dimensiones de la calidad de vida como separadas, ya que éstas interactúan, se influyen y se forman entre sí, por lo que es necesario verlas como interconectadas e interdependientes. En la formulación de estas categorías densas e integradoras se tomó como referencia, más que otros estudios sobre la calidad de vida en entornos urbanos - realizados y formulados en relación con otros contextos temporales y espaciales-, las narrativas de los habitantes. De acuerdo con Arfuch (2010, pp. 87-89), toda experiencia de vida se inscribe en la narrativa, y ésta a su vez se inscribe en una temporalidad. Las narrativas articulan, integran y contextualizan las experiencias individuales y colectivas de los sujetos, tanto directas como indirectas, es decir, tanto experiencias personales, como aquellas conocidas sobre situaciones vividas por familiares, amigos, vecinos, etcétera (Polkinghorne, 1988; Bertaux, 2011).

De acuerdo con lo anterior, las categorías integradoras fueron:

a) Vivienda anterior y vivienda actual

b) Condiciones del fraccionamiento y su impacto en la vida cotidiana

c) Interacciones sociales y uso del espacio público.

d) Satisfacción y expectativas con la vivienda y el fraccionamiento.

Estas categorías formaron parte de la construcción de un modelo dinámico e interpretativo de la calidad de vida, en la que el habitante esta al centro de una realidad concreta y localizada: la periferia metropolitana de Morelia, Michoacán (Figura 1).

\section{FIGURA 1}

Ubicación del Fraccionamiento Villas del Pedregal.

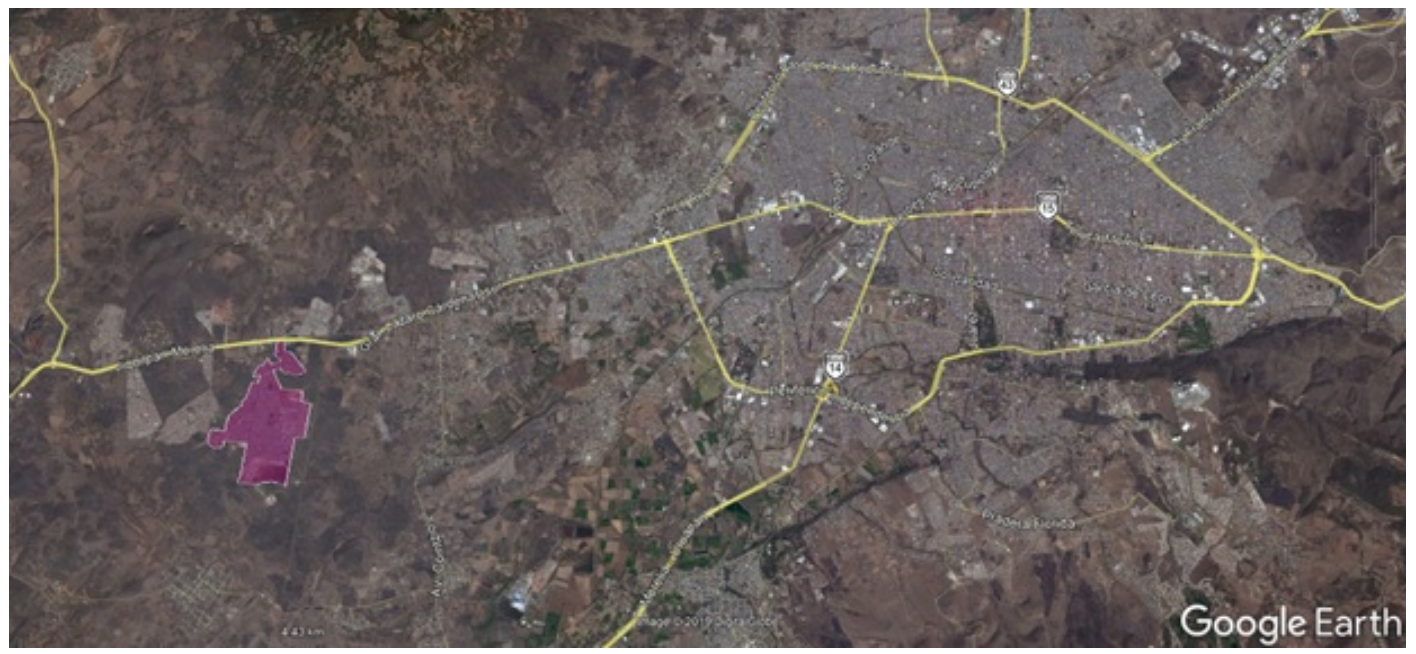

El Fraccionamiento Villas del Pedregal (color violeta) está ubicado en la periferia de la zona metropolitana de Morelia -Michoacán, México-, a una distancia de $15 \mathrm{~km}$ del anillo periférico (color amarillo) de la ciudad de Morelia. Elaboración propia sobre la base de Google Earth pro 


\section{A. Vivienda anterior y vivienda actual.}

Fue común que los habitantes entrevistados recrearan narrativamente la experiencia de dejar la vivienda anterior y llegar a habitar su vivienda actual. En este contexto fue recurrente que se compararan las viviendas y se narraran conflictos entre las experiencias vividas en ambas (Kohler, 1993). Por ejemplo, al preguntarles en las entrevistas sobre si las dimensiones de su actual vivienda le parecían adecuadas, muchas veces respondían que su vivienda anterior era más grande y narraban el uso que hacían de esos espacios, así como las dificultades de realizar ciertas actividades habituales en la nueva vivienda. Los habitantes entrevistados narraron también las diferencias en calidad y cantidad de los equipamientos, infraestructura y servicios urbanos, así como los problemas a los que se enfrentaron en ambos entornos habitacionales. La situación del individuo y su familia cuando llegan a vivir a un entorno periférico, que no cuenta con todos los equipamientos necesarios, en donde no conocen a los vecinos y no tienen la certeza de que vayan a mejorar las condiciones del fraccionamiento -y en general sus condiciones de vida-, se refleja en sus narrativas. El habitante al narrar enlaza sus experiencias pasadas con las presentes y expresa sus expectativas a futuro, lo que denota sus maneras de habitar.

\section{B. Relaciones vecinales y uso del espacio público.}

La imposibilidad de elegir en dónde vivir, con quién vivir y en qué tipo de vivienda se ha convertido en un eje de malestar cotidiano. No todos los habitantes de la periferia residen allí por libre elección, lo hacen porque esas son las viviendas que tienen la capacidad de obtener; esto de ninguna manera garantiza que los residentes se encuentren satisfechos con su vivienda, ni con sus vecinos, ni tampoco es garantía de que lo hagan con el espacio público y el entorno habitacional. De acuerdo con la información recabada en el trabajo de campo y su interpretación, dimos cuenta de que en el caso del Fraccionamiento Villas del Pedregal los espacios públicos realmente no funcionan como escenario de convivencia, $y$, menos aún -como comenta Borja (2003, p. 176) -, como el escenario de integración social que todo espacio público debería de ser. Con ello no se está valorando apriori a todo espacio público, ni dando por supuesto que en todas las periferias metropolitanas existe insatisfacción con el mismo (Duhau y Giglia, 2008, pp. 194-195); nuestra valoración se da en relación con el trabajo de campo realizado y con la forma que fue adoptando, de mayor inmersión por experimentar personalmente el ambiente vecinal.

\section{Condiciones del fraccionamiento y su impacto en la vida cotidiana.}

Habitar significa reconocer el lugar, reconocerse en él, habitar-se (Del Acebo, 1996, p. 202). Habitar es un enorme repertorio de experiencias, prácticas, tácticas y estrategias de cada individuo para expresar y exteriorizar su modo de vida. Así que la construcción de la narrativa sobre el habitar, los habitantes la construyen en la interacción con el entrevistador, y en la presencia física en su vivienda y fraccionamiento. De esta manera, el análisis no se fundamenta en el discurso único e independiente del entrevistador, sino también en la relación que es establecida con el habitante y el lugar. El lugar encarna las experiencias y aspiraciones de quienes lo habitan, y es a partir de la perspectiva de los sujetos que éste debe comprenderse (Tuan, 1979, p. 378). De esta manera, la vivienda como lugar para habitar y el entorno urbano donde se localiza exhiben su propio carácter discursivo en sus espacios públicos y privados, sus calles, áreas verdes, equipamientos, etc. Estos espacios constituyen tramas simbólicas en constante movimiento, que cambian sus significados con los acontecimientos, prácticas y demás eventos que los habitantes experimentan cotidianamente. 


\section{Satisfacción y expectativas con la vivienda y el fraccionamiento}

La vivienda está cargada de un cúmulo de símbolos y significados y, más allá de cumplir la función de alojamiento, significa protección y resguardo para quienes la habitan (Bachelard, 2000), mercancía proveedora de estatus social y progreso (Harvey, 2012). Significa también el lugar para habitar (Tuan, 2001) y para formar un hogar (Pezeu-Massabuau, 1988, p. 54). Por todo eso, la vivienda es tanto el medio necesario para alcanzar calidad de vida, como también un obstáculo para lograrlo en caso de no reunirse las condiciones de habitabilidad que los habitantes necesitan, desean y anhelan. Con base en la información recabada para esta categoría integradora de la calidad de vida se descifraron, a partir de las narrativas de los habitantes, las dimensiones significativas en torno a su vivienda y entorno habitacional, dadas por las necesidades y deseos satisfechos e insatisfechos de los habitantes, así como las expectativas a futuro que ellos se han forjado en relación con esto. Las experiencias de habitar, ya sean positivas o negativas, forman parte del pasado y presente del habitante; en el "¡cómo ha sido?" y en el "¿cómo es habitar una vivienda y un entorno habitacional periférico?" se proyecta el “¿cómo podría ser?”, porque cada individuo es producto de un proceso social en curso (Mead, 1968), en el que el "convertirse en" o "llegar a ser" se incorporan a sus experiencias, acciones y prácticas. Así, los modos de habitar la vivienda van más allá de la simple realización de actividades en ésta, y reúnen los significados, sentidos, experiencias y expectativas de quienes la habitan.

En resumen, el principio metodológico de la investigación se encuentra en las narrativas de los habitantes, resultado de los recorridos de observación (1) y su cruce analítico con las entrevistas semiestructuradas (2), las cuales se fortalecieron con la observación participante (3), ya que la misma permitió la inmersión más profunda en el lugar y en la vida de los habitantes a través de la experiencia de habitar del investigador. Con base en la interdependencia mutua de todos los instrumentos, finalmente se aplicó el cuestionario de la encuesta (4), con la finalidad de completar la información obtenida por los otros instrumentos, y ratificar que se poseía la información más pertinente de acuerdo a las narrativas de los habitantes (Figura 2).

FIGURA 2

Esquema del diseño de la investigación

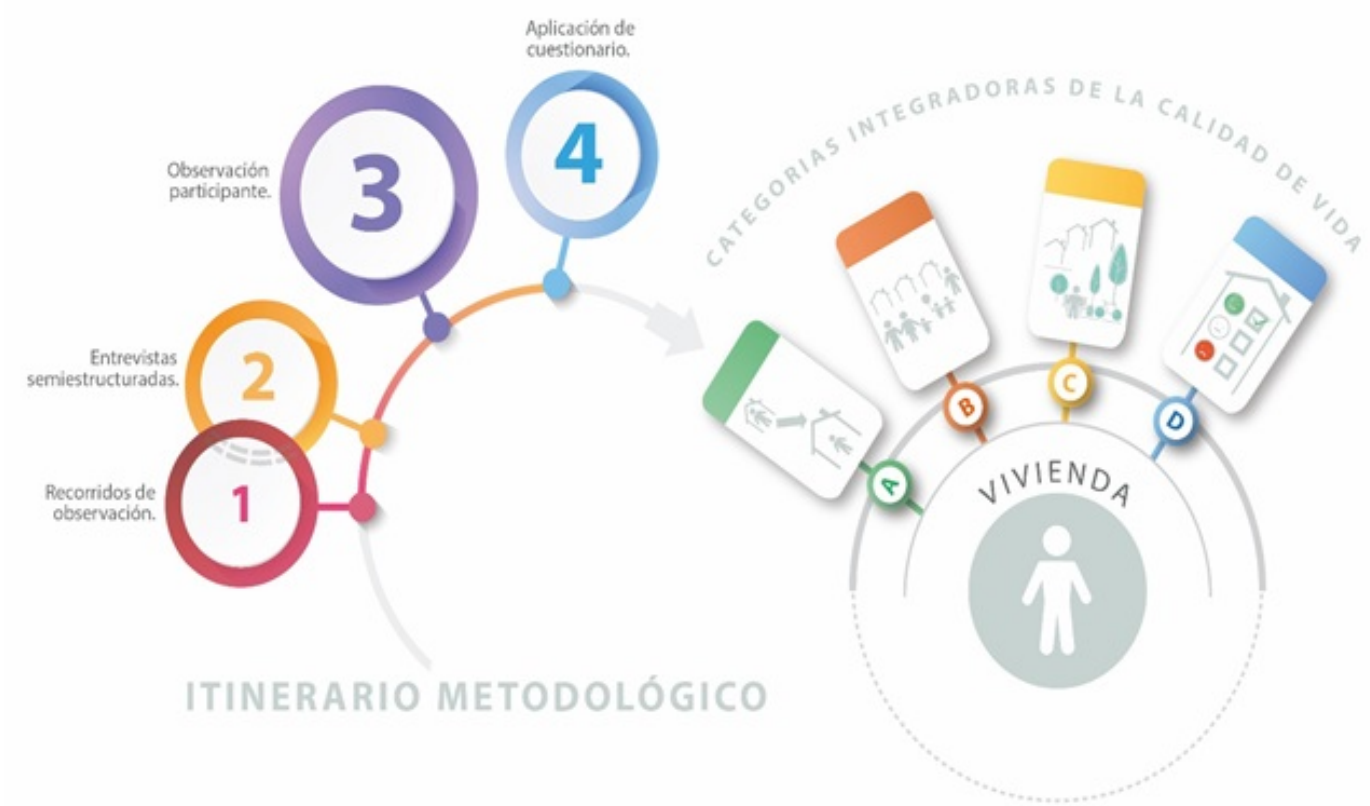

La integración y cruce analítico de la información, producto del itinerario de investigación llevado a cabo, nos permitió valorar a través de las categorías integradoras: a) Vivienda anterior y vivienda actual; b) relaciones vecinales y uso del espacio público; c) condiciones del fraccionamiento y su impacto en la vida cotidiana; d) satisfacción y expectativas con la vivienda y el fraccionamiento; la calidad de vida de los habitantes desde su propia perspectiva. Elaboración propia. 
Las mencionadas categorías se integran a partir del habitante en su experiencia de habitar su vivienda y entorno habitacional. Éstas no se analizaron de manera aislada una de otra, no solo porque tienen vínculos de interdependencia entre ellas y con el habitante, sino además porque cada una de ellas adquiere su identidad propia en contrapunto con las demás.

\section{RESULTADOS Y DISCUSIóN}

El resultado de la investigación pretende ser una herramienta para el estudio de la vivienda y la calidad de vida de los habitantes de la periferia metropolitana. Se busca también que la realidad que se narra sirva para extrapolarse a otras realidades, en donde quizá tenga que haber nuevas preguntas y respuestas, acompañadas de sus respectivos ajustes, que no por ello dejen de lado dimensiones compartidas. En este texto no se presentan datos duros, sino una interpretación de un itinerario metodológico de un trabajo de investigación. En este itinerario, las técnicas de investigación se ejecutaron en diferentes días y horarios durante un año completo, con el fin de atribuir las diferentes condiciones que se presentan en la vida cotidiana, como las lluvias, inundaciones, el calor, el frío, el día, la noche, las festividades, los días sin agua potable, los actos delictivos, entre otros eventos que influyen en los modos de habitar de los habitantes y en la valoración que sobre su calidad de vida tienen en ese entorno habitacional. La forma que fue adoptando el trabajo de campo buscó no tener una observación parcial, lo que se logró a partir de la conexión de las prácticas cotidianas y las subjetividades en diferentes espacios y tiempos. Se trata de un planteamiento que permite pensar la investigación en términos relacionales, y, en lugar de mostrar la realidad estática, permite entrar en un entramado de conexiones y experiencias de habitar que cambian constantemente, por ejemplo, después de que un habitante sufriera un asalto o se enterase enterado de que un vecino había sido asaltado, la percepción del miedo se expande entre los vecinos, y las prácticas de los habitantes se modifican, ya que, aunque el miedo se constituye como una experiencia individualmente experimentada, es socialmente construida y culturalmente compartida (Reguillo, 2008, p. 70).

$\mathrm{Al}$ miedo se le asigna horario, porque el espacio tiene atributos en función del tiempo, el día, la noche, los días de trabajo, los días de descanso, los días lluviosos, los de inundaciones, entre otros, que modifican los usos del espacio. En la noche, el espacio se percibe como peligroso y con las inundaciones como intransitable. Es este paso del tiempo y sus eventos lo que propicia la interacción social entre los individuos, así como la interacción entre ellos y el espacio (Muñoz, 2006).

Otro potencial de la investigación fue la intercalación de las técnicas de investigación, es decir, se pudo probar, con base en los recorridos de observación, la pertinencia del guion de la entrevista, y se identificó, con base en las narrativas de las entrevistas, en qué aspectos era más pertinente concentrar la observación. Aun cuando indudablemente se requirió de una situación de entrevista para obtener las narraciones de los habitantes, la narrativa se descifró desde la articulación de la observación participante en relación con el espacio y en la interacción con los habitantes. Como señala Spradley (1980, p. 3), más que estudiar a la gente, se aprende de ella, y de esa manera se puede captar el significado que le atribuyen a sus prácticas y objetos. Este dinamismo en el proceso del trabajo de campo permite al investigador ser partícipe en los ámbitos y en las prácticas en donde se desarrollan y constituyen los modos de habitar. Así, se experimenta el espacio al tiempo que se da cuenta de las posibilidades y dificultades que tienen los habitantes para acceder a equipamientos, servicios, para relacionarse con los vecinos, para desplazarse desde la periferia lejana, etcétera. De esta manera fue que, a partir de la propia experiencia espacial del investigador, se conocieron las de los investigados, en un proceso definido por Ruiz Olabuénaga (2012) como investigación en-de-con. Es así que el experimentar el espacio a partir de las prácticas y acciones tendientes a satisfacer las necesidades de habitar hace posible comprender la valoración de la calidad de vida de los habitantes con relación a las condiciones de los entornos habitacionales en que viven. 


\section{Conclusiones}

Este trabajo es producto del análisis reflexivo de experiencias de trabajo de campo. El partir de una mirada retrospectiva del proceso de investigación en su conjunto permite generar problematizaciones en relación con los estudios sobre la vivienda y la calidad de vida, que son de relevancia para la comprensión de las actuales realidades metropolitanas de Latinoamérica. Los debates teóricos y metodológicos sobre los problemas urbanos y metropolitanos requieren profundizar en las transformaciones en el hábitat y en las formas de habitar a partir de las acciones de las empresas inmobiliarias, y de las políticas, normativas y estrategias urbanas, cuyos impactos abarcan y rebasan los centros urbanos consolidados e incluyen periferias metropolitanas caracterizadas por tener déficits en cuanto el abastecimiento de equipamientos, servicios urbanos, espacios públicos y viviendas de calidad, que impactan negativamente en la valoración que los habitantes tienen sobre su calidad de vida.

Así, a partir de un intenso trabajo de campo se construyó un modelo interpretativo que pretende ser una herramienta para estudiar la calidad de vida de quienes habitan en entornos habitacionales en las periferias metropolitanas, a partir de las articulaciones e interacciones entre el individuo y la colectividad, lo material e inmaterial, así como entre escalas espaciales, la vivienda, el fraccionamiento, el entorno urbano y metropolitano. La discusión planteada a lo largo de este texto permite señalar que los geógrafos humanos, al igual que todos los científicos sociales, no pueden dejar de pensar reflexivamente en cómo hacer trabajo de campo para acercarse a la comprensión del punto de vista del sujeto. Solo así es posiblecomprender los territorios donde habitan, con sus múltiples búsquedas, necesidades, intereses y expectativas de vida.

Los habitantes aprenden cuáles son los espacios peligrosos y cuándo es prudente ya no salir de su vivienda; aprenden dónde comprar diferentes productos, aprenden a trasladarse desde las periferias y a sortear las dificultades que se les presentan cotidianamente por las condiciones del fraccionamiento; aprenden, pues, a satisfacer sus necesidades y deseos en su vivienda y fraccionamiento con relación a sus experiencias espaciales (Claval, 1999). El espacio como experiencia está integrado por lo imaginario, que se alimenta del futuro, y la memoria, que lo hace del pasado. El espacio como experiencia se estructura por narrativas, a la vez que participa en la estructuración de los sujetos que narran; así el sujeto expresa narrativamente su experiencia espacial en relación con su familia, amigos, vecinos, compañeros de trabajo, entre otros sujetos, partícipes en mayor o menor medida de la misma (Lussault, 2015, pp. 24-25). Por ello, "la Geografía no se puede limitar a tomar en cuenta a los grupos sociales, también debe anclarse en el sujeto, el individuo, la persona, el actor” (Di Meo y Buléon, 2005, p. 39 citado en Lindón, 2008, p. 10).

Las narrativas de los habitantes se fueron construyendo gradualmente a la luz de la información procedente de los recorridos de observación, de las entrevistas, de la observación participante y de la aplicación del cuestionario de la encuesta, lo que permitió comprender sus experiencias espaciales, al igual que las de los individuos en esa interacción dialógica (Elias, 1990). Partir de las narrativas y las prácticas cotidianas de los habitantes y de su relación con el espacio que habitan es promover una visión egocéntrica en el estudio de las metrópolis. Como sugiere Tuan (2005, p. 160): "Ponerse en la piel de las otras personas, beber de la experiencia vital y las creencias de la gente de a pie" nos permitirá articular un entendimiento integral de los factores que intervienen en la constitución y praxis del principal objeto de estudio de la Geografía, el espacio en su relación con el sujeto y la sociedad que lo transita, habita, significa y se significa en él. 


\section{REFERENCIAS}

Allardt, E. (1996). Tener, amar, ser: una alternativa al modelo sueco de investigación sobre el bienestar. En A. Sen y M. Nussbaum (comps.), La calidad de vida (pp. 126- 134). México: Fondo de Cultura Económica.

Arfuch, L. (2010). La vida como narración. En L. Arfuch, El espacio biográfico. Dilemas de la subjetividad contemporánea. Buenos Aires: Fondo de Cultura Económica.

Bachelard, G. (2000). La poética del espacio. México: Fondo de Cultura Económica

Bertaux, D. (2011). El enfoque biográfico: su validez metodológica, sus potencialidades. Acta Sociológica, 56, 61-93

Blumer, H. (1969). Symbolic Interactionism: Perspective and Method. California: University of California Press, New Jersey: Hall Englewood Cliffs.

Blunt, A. (2005). Cultural geography: cultural geographies of home. Progress in Human Geography, 29(4), 505-515. https://doi.org/10.1191/0309132505ph564pr

Blunt, A., y Dowling, R. (2006). Home (Key Ideas in Geography). Londres: Routledge.

Borja, J. (2003). La ciudad conquistada. Madrid: Alianza Editorial.

Borja, J. (2017). Contra la injusticia espacial, por los derechos ciudadanos, la igualdad y la sostenibilidad. Folios, 32, 4-21. Recuperado de http://www.revistafolios.mx/hemeroteca/32_0

Brickell, K. (2012). 'Mapping' and 'doing' critical geographies of home. Progress in Human Geography, 36(2), 225-244. https://doi.org/10.1177/0309132511418708

Claval, P. (1999). Los fundamentos actuales de la geografía cultural. Documents d'Análisi Geográfica, 34(1), 25-40.

Collignon, B. (2010). De las virtudes de los espacios domésticos para la geografía humana. En A. Lindón y D. Hiernaux (Dir.), Los giros de la Geografía Humana. Desafios y horizontes (pp. 201 - 215). Barcelona: Anthropos Editorial; México: UAM-Iztapalapa

Cortés Alcalá, L. (1996). La cuestión residencial: basespara una Sociología del Habitar. Madrid: Editorial Fundamentos. Cresswell, T. (2004). Place: a short introduction. Oxford: Blackwell

Cuenya, B. (2012). Grandes proyectos como herramientas de creación y recuperación de plusvalías urbanas: Ejemplos de argentina y Brasil. En A. Ziccardi (Coord.), Ciudades del 2010: Entre la sociedad del conocimiento y la desigualdad social (pp. 1019-1038). México: Universidad Nacional Autónoma de México.

De Certeau, M. (1996). La invención de lo cotidiano 1. Las artes del hacer. México: Universidad Iberoamericana.

Del Acebo, I. E. (1996). Sociología del arraigo: una lectura critica de la teoría de la ciudad. Buenos Aires: Claridad.

Diener, E. (2006). Guidelines for national indicators of subjective well -being and ill- being. Applied Research in Quality of life, 1(2), 151 - 157. https://doi.org/10.1007/s11482-006-9007-x

Duhau, E., y Giglia, A. (2008). Las reglas del desorden: habitar la metrópoli. México: Siglo XXI. UAM-Azcapotzalco.

Elias, N. (1990). La sociedad de los individuos. Barcelona: Península.

Espinosa, F. (2015). Vivienda de interés social y calidad de vida en la periferia de la ciudad de Morelia Michoacán. México: Universidad Nacional Autónoma de México, Infonavit.

García Ramón, M. D. (1999). Canvi o continuitat en la geografla cultural? Notes a l'entorn de Cultural Turns/ Geographical Turns. Documents d'Análisi Geográfica, 34(1), 135-140

Giddens, A. (1995). La constitución de la sociedad. Bases para la teoría de la estructuración. Buenos Aires: Amorrortu Editores

Harvey, D. (2012). Rebel Cities. From the Right to the City to the Urban Revolution. Londres, Nueva York: Verso

Hiernaux, D., y Lindón, A. (2004). Repensar la periferia: De la voz a las visiones exo y egocéntricas. En A. Aguilar (coord.), Procesos metropolitanos y grandes ciudades: Dinámicas recientes en México y otros países (pp. 413-443). México: Universidad Nacional Autónoma de México. Miguel Ángel Porrúa. 
Hiernaux, D. (2008) Geografía Objetiva Versus Geografía Sensible: Trayectorias divergentes de la geografía humana en el siglo XX. Revista da ANPEGE, 4(4), 27-38. Recuperado de http://ojs.ufgd.edu.br/index.php/anpege/ar ticle/view/6597/3597

Kohler, R. C. (1993). Narrative Analysis. Newbury Park, CA: Sage.

Lindón A., y Hiernaux, D. (Dirs.) (2010). Los giros de la Geografia Humana. Desafios y horizontes. Barcelona: Anthropos Editorial; México: UAM-Iztapalapa

Lindón, A. (2005). El mito de la casa propia y las formas de habitar. Scripta Nova. Revista Electrónica de Geografía y Ciencias Sociales. vol IX 194(20), (s/n). Recuperado de http://www.ub.es/geocrit/sn/sn-194-20.htm

Lindón, A. (2006). La casa bunkery la deconstrucción de la Ciudad. Liminar: Estudios Sociales y Humanisticos, 4(2), 18-35. Recuperado de http://liminar.cesmeca.mx/index.php/r1/article/view/208/191

Lindón, A. (2008). De las geografías constructivistas a las narrativas de vida espaciales como metodologías geográficas cualitativas. Revista da ANPEGE, 4(4), 3-27. Recuperado de http://ojs.ufgd.edu.br/index.php/anpege/article /view/6596

Lindón, A. (2009). La construcción socio-espacial de la ciudad. El sujeto-cuerpo y el sujeto sentimiento. Revista latinoamericana sobre Cuerpos, Emociones y Sociedad. 1(1), 6-20. Recuperado de http://www.relaces.com.ar/in dex.php/relaces/issue/view/1/showToc

Lussault, M. (2015). El Hombre espacial. La construcción social del espacio humano. Madrid: Amorrortu

Mallett, S. (2004). Understanding home: a critical review of the literature. The Sociological Review, 52(1), 62 - 89. $\mathrm{h}$ ttps://doi.org/10.1111/j.1467-954X.2004.00442.x

Mead, G. H. (1968). Espiritu, persona y sociedad. Desde el punto de vista del conductismo social. Barcelona: Paidós

Muñoz, F. (2006). El tiempo del territorio, los territorios del tiempo. En J. Nogue y J. Romero. Las otras Geografias (pp. 235-254). Barcelona: Tirant lo Blanch.

Pezeu-Massabuau, J. (1988). La vivienda como espacio social. México: Fondo de Cultura Económica.

Philo, C. (1999). Más palabras, más mundos: Reflexiones en torno al giro cultural y a la geografía social. Documents d'Analisi Geografica, (34), 81-99.

Polkinghorne, D. E. (1988). Narrative knowing and the human sciences. Albany, NY: State University of New York Press.

Reguillo, R. (2008). Sociabilidad, inseguridad y miedos: trilogía para pensar la ciudad. Alteridades, 18(36), 63-74.

Ruíz Olabuénaga, J. I. (2012). Metodología de investigación cualitativa. Bilbao: Universidad de Deusto.

Scanlon, T. M. (1996). El valor, el deseo y la calidad de vida. En A. Sen y M. Nussbaum (comps.), La calidad de vida (pp. 245-264). México: Fondo de Cultura Económica.

Seamon, D. (1979). A geography of the lifeworld: Rest and encounter. Nueva York: St. Martin's Press.

SEDATU y CONAVI (2017). Código de Edificación de Vivienda, 3a edición, México: International Finance Corporation. World Bank Group. IFC; Organismo Nacional de Normalización y Certificación de la Construcción y Edificación. Recuperado de https://www.gob.mx/conavi/documentos/codigo-de-edificacion-d e-vivienda-3ra-edicion-2017

Silva, A. (2013). Imaginarios, el asombro social. Bogotá: Universidad Externado de Colombia.

Spradley, J. P. (1980). Participant Observation. Nueva York: Holt, Rinehart and Winston.

Tuan, Y. F. (1979). Space and place: humanistic perspective. En S. Gale y G. Olsson (Ed.), Philosophy in Geography (pp. 387-487). Dordrecht: Springer Netherlands.

Tuan, Y. F. (2001). Space and Place: The Perspective of Experience. Minneápolis: University of Minnesota.

Tuan, Yi-Fu (2005). Hogar y Cosmos. Un punto de vista cosmopolita. Barcelona: Melusina

Tuan, Yi-Fu (2007). Topofilia. Un estudio de laspercepciones, actitudes y valores sobre el entorno. Santa Cruz de Tenerife: Melusina. 
Warf, B., y Arias, S. (2008). The Spatial Turn: Interdisciplinary Perspectives. Nueva York: Routledge.

Ziccardi, A. (2016). Vivienda, gobiernos locales y gestión metropolitana. En A. Ziccardi y D. Cravacuore (coords.), Los gobiernos locales y las politicas de vivienda en México y América Latina (pp. 13-30). Buenos Aires: Ediciones Centro Cultural de la Cooperación Floreal Gorini; Quilmes: Universidad Nacional de Quilmes; México: Universidad Nacional Autónoma de México.

\section{Notas}

1 Fraccionamiento es la división de un terreno en manzanas y lotes, que requiera del trazo de una o más vías públicas, así como de la ejecución de obras de urbanización que le presten servicios urbanos (SEDATU y CONAVI, 2017, p. 34).

2 En este trabajo, más que mostrar cifras y datos concretos, se buscó explorar y reflexionar sobre el itinerario metodológico de la investigación que durante años de trabajo de campo en la periferia metropolitana de Morelia Michoacán, México, se ha realizado. Posteriormente, a partir del itinerario se formularon reflexiones sobre los vínculos analíticos existentes entre la vivienda social y la calidad de vida de los habitantes de la periferia metropolitana de Morelia, Michoacán, México, que bien pueden ser referencia, se pueden indagar y/o complementar para el caso de estudio de otras periferias metropolitanas de Latinoamérica. 